\title{
THE EFFECT OF PLATELET RICH FIBRIN ON ANGIOGENESIS DURING PERIODONTAL REGENERATION
}

\author{
Moody A. Samuel ${ }^{1} B D S$, Maha A. Abou $\operatorname{Khadr}^{2} P h D$, Azza S. Koura ${ }^{3} P h D$, \\ Marwa I. Madi ${ }^{4} P h D$
}

\begin{abstract}
INTRODUCTION: Platelet rich fibrin (PRF) is an autologous healing biomaterial composed of a fibrin clot matrix entrapping leukocytes, cytokines, living progenitor cells, and platelets capable of releasing various growth factors harvested from a simple blood sample.

OBJECTIVES: The aim of this experimental study was to evaluate the effect of PRF on angiogenesis during the periodontal regeneration of surgically created grade II furcation defects in dogs.

MATERIALS AND METHODS: A split mouth design was carried out using the third and fourth mandibular premolars of 2 healthy mongrel adult dogs. A total of eight grade II furcation defects were surgically created. The defects in the study side were augmented with PRF mixed with a $\beta$-TCP and covered by a PRF membrane and a collagen membrane. In the control side the defects were augmented with $\beta$-TCP and covered with a collagen membrane. The dogs were sacrificed after 1 and 2 months. Samples were dissected and prepared for histological evaluation.

RESULTS: Histological results showed that PRF addition led to higher vascularization of the study samples at all age groups; this was evident at one month in the form of numerous blood vessels entrapping RBCs and at 2 months by the presence of red bone marrow and blood vessels
\end{abstract} entrapping RBCs within.

CONCLUSIONS: The addition of PRF to $\beta$-TCP was found to be effective in enhancing angiogenesis during the periodontal regeneration of grade II furcation defects.

KEYWORDS: Platelet Rich Fibrin, Angiogenesis, Periodontal regeneration, Grade II furcation defects, $\beta$-TCP.

1- Bachelor of Dental medicine and surgery 2007, Faculty of dentistry, Alexandria University, Egypt.

2- Professor of Periodontology, Alexandria University, Egypt.

3- Assistant Professor of Oral Biology, Faculty of Dentistry, Alexandria University, Egypt.

Lecturer of Periodontology, Faculty of Dentistry, Alexandria University, Egypt.

\section{INTRODUCTION}

Angiogenesis is the formation of new blood vessels inside the wound. It underlies the success of tissue healing and regeneration, as the newly formed blood vessels are crucial in the delivery of oxygen, nutrients, and important cells from nearby tissues in the hypoxic microenvironment of healing wounds (1). This is essential in the healing of long bones as demonstrated by Wang et al; (2) as they observed that the improving of angiogenesis by shock waves remarkably improves the union of fractured bone. In another study Wang et al; (3) observed the critical role of vascular endothelial growth factors (VEGF) in the regulation of angiogenesis and osteogenesis in the healing of bone fractures in rabbits.

Various growth factors are stored in platelets and are released upon platelet activation giving the platelets a crucial role in wound healing and regeneration of injured tissues besides their haemostatic functions. Thus the use of platelets concentrates was proposed to promote tissue healing and periodontal regeneration (4). Two generations of platelet concentrates were used to enhance periodontal regeneration. The first generation includes platelet rich plasma (PRP) production protocols, while the second generation is the platelet rich fibrin (PRF) (5).

PRF was first developed by Choukroun (6) in 2001 who defined PRF as an autologous healing biomaterial made of a matrix of an autologous fibrin clot entrapping leukocytes, cytokines, living progenitor cells, and platelets capable of releasing various growth factors harvested from a simple blood sample.
Unlike PRP, PRF is obtained through a natural polymerization process without the addition of anticoagulants, giving a chance for a dense fibrin clot formation entrapping platelets and leukocytes. The platelets are also activated in a natural mechanism without the need of brutal activation with bovine thrombin, as with most PRP protocols, thus releasing growth factors and cytokines such as Platelet-derived growth factor (PDGF), vascular endothelium growth factor (VEGF), transforming growth factor-beta (TGF- $\beta$ ), insulin-like growth factors (IGF), endothelial Growth factors family (EGF), and brain-derived neurotrophic factor (BDNF) in a slow releasing mechanism during a period of 7 days or more after which the fibrin clot is disintegrated naturally by fibrinolysis (7). Among the various growth factors released by platelets during the wound healing process VEGF were found responsible for regulating angiogenesis (3).

The aim of this experimental study was to evaluate the effect of PRF on angiogenesis during the periodontal regeneration of surgically created grade II furcation defects in dogs.

\section{MATERIAL AND METHODS}

Care and use of experimental animals was undertaken in accordance to the guidelines set by the ethics committee, faculty of dentistry, Alexandria university.

Animal Selection: A total of 2 healthy adult mongrel dogs (Canis familiaris) about 18 to 24 months old and weighing between 18 to $24 \mathrm{~kg}$ were included in this study. The dogs had good systematic health with no gingival inflammation, and showed intact maxillary and mandibular teeth. The 
animals were adapted to the housing conditions 4 weeks before the study.

Study design: A split mouth design was applied in this study. A total number of 8 surgically created acute type critical -sized grade II furcation defects were induced in the mandibular third (PM3) and fourth (PM4) premolars on both sides of each dog. The defects were divided into two groups each compromising 4 defects as follows:

- Control group: the created defects where treated by using alloplast and then covered by a collagen membrane.

- Study group: the created defects where treated by using alloplast mixed with PRF cuts and covered by a PRF membrane then by a collagen membrane.

\section{Materials:}

Bone substitute material (Alloplast): (Genesis BCP ( $\left.{ }^{\mathrm{TM}}\right)$ ) by Dio-implant, Korea) A biphasic calcium phosphate composed of Hydroxyapatite $(60 \%)$ and Beta-Tri-CalciumPhosphate (Beta-TCP) (40\%). With 70\% macropores (100 to $500 \mu \mathrm{m})$ and $30 \%$ micropores $(\leq 10 \mu \mathrm{m})$.

Collagen Membrane: (Biocollagen collagen membrane by BioTECK, Italy) Type 1 lyophilized equine collagen from Achilles tendon ( $25 \times 25 \times 0.2 \mathrm{~mm})$.

Platelet Rich Fibrin (PRF): PRF was prepared as follows (8): About $10 \mathrm{ml}$ of venous blood was drawn from the cephalic vein of each dog, and immediately divided into 2 plains (anticoagulant free) glass blood collection tubes each of $5 \mathrm{ml}$. Both tubes were centrifuged immediately. Blood samples were centrifuged using a tabletop centrifuge for 10 min at $3000 \mathrm{rpm}$. (Figure 1)

After centrifugation, the resultant blood samples were divided into three layers:

- The top most layer consists of acellular platelet poor plasma.

- The middle layer consists of PRF or fibrin clot.

- The bottom layer consists of Red blood cells.

Each fibrin clot was gently removed from the test tube and any excess was removed by using sharp scissor. One fibrin clot was cut into small pieces and mixed with the bone graft substitute material in order to fill the 2 created defects in the test side. The other fibrin clot was used to obtain the PRF membrane. The fibrin clot was gently squeezed between two sterile pieces of gauze soaked in saline thus to squeeze out the serum and form the PRF membrane. The resultant membrane was further cut into two halves with a pair of sharp scissors to cover the 2 created defects in the test side.

Surgical procedures: All surgical procedures were performed under general anesthesia using intramuscular injection of $0.1 \mathrm{ml}$ ketamine hydrochloride (Ketamine10\%® by Alfasan, Holland.) and $0.05 \mathrm{ml}$ xylazine hydrochloride (Xyla-ject巴 by Adwia co. S.A.E., Egypt.), for each 100mg body weight.

\section{Creation of Grade II furcation defects:}

Infiltration anesthesia using $2 \%$ xylocaine/epinephrine (Artinibsa ${ }^{\circledR}$ by Inibsa, Spain.) was administered at the surgical site. Sulcular incisions were performed using Bard Parker blade number 15 , and then a mucoperiosteal flap extending from PM2 to M1 was elevated. The alveolar bone buccal to the furcation area was removed using carbide burs on a low speed micro-motor under copious saline irrigation to expose the roots of the experimental teeth. Then grade II critical sized furcation defects were created in the furcations of PM3 and PM4on either sides of the mandible. The dimensions of the defects were measured using a periodontal probe with Michigan O with Williams' calibration (Kohler ( ) Medizintechnik GmbH \& Co., Germany) the dimensions of the resultant critical size created grade II furcations defects were as follows: A $5 \mathrm{~mm}$ in the vertical component (distance from the furcation fornix to the base of the defect) and $4 \mathrm{~mm}$ in the horizontal component (distance from the buccal surface to the internal wall of the furcation). Root planing was performed on all exposed root surface using manual curettes. After the creation of the defects was completed, reference notches were created on both roots (at the most apical part of each root in relation to the base of the defect) to determine the region of interest (ROI) (9).

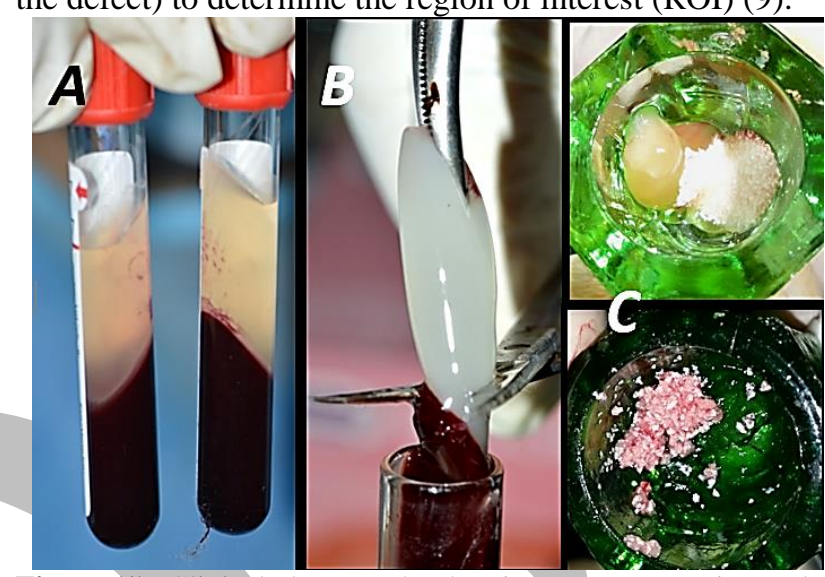

Figure (1): Clinical photographs showing PRF preparation and mixing with alloplast.

A. After centrifugation three layers are formed with PRF clot in the middle portion.

B .PRF clot separation,

C. PRF mixing with Alloplast.

Management of the defects: Root conditioning was carried out using tetracycline (Tetracid $®$ by Chemical industries development CID, Egypt) soaked cotton pellets in order to modify the root surface to enhance connective tissue reattachment. In the control side (left side), the defects were augmented with alloplast and then covered by collagen membrane (Figure 2). While. In the study side (the right side) the defects were augmented with Alloplast mixed with PRF cuts and serum exudates then covered with PRF membrane and collagen membrane. (Figure 3)

Histological procedures The specimens were decalcified in $8 \%$ trichloroacetic acid and processed to obtain 5 micron thick sections. These sections were stained with Harris Hematoxylin and Eosin Stain (H\&E) for general examination and evaluation of healing (10) and with Gomori's trichrome stain to examine the new bone formation and collagen organization (11).

\section{Histological Results}

At one month, both the study and the control samples showed the old bone trabeculae at the base of the defect covered by new bone trabeculae formation extending towards the bifurcation area while the rest of the defect was filled with granulation tissue. A distinct histological feature found in the study group specimens is the high vascularization evident by the presence of multiple blood vessel formation found in the study samples (Figure 3).

At two months, both groups showed excellent bone fill with thick bone trabeculae, the bone trabeculae in both groups showed resting lines and abundant osetocytes with a lining osteoblastic layer. PDL fibers in both groups were well oriented containing many fibroblasts. However, the study group specimens showed higher evidence of 
vascularization by the presence of red bone marrow and more blood vessels entrapping RBCs within. (Figure 4)

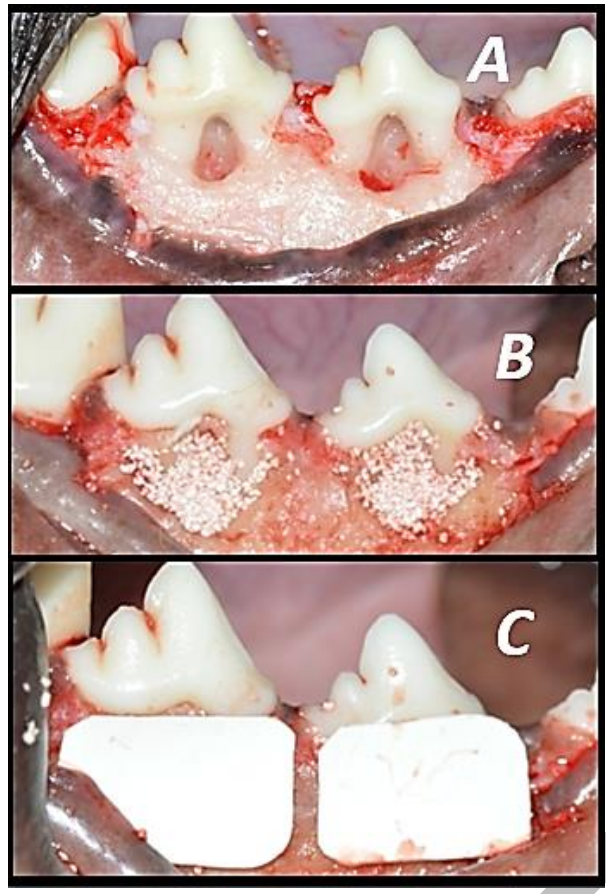

Figure (2): Clinical photographs showing

A. The created furcation defects.

B. Management of the control side in which the defects were augmented with alloplast.

C. The defects covered by a collagen membrane.

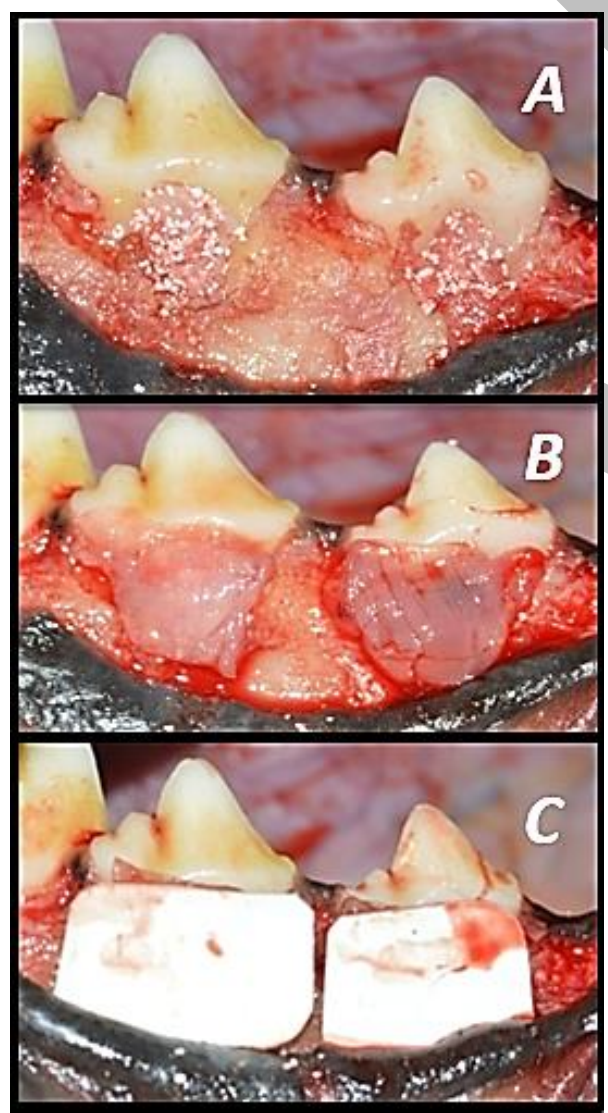

Figure (3): Clinical photographs showing

A. Management of the study side in which the defects were augmented with Alloplast mixed with PRF cuts and serum exudates.

B. Defect covered with PRF membrane.

C. Collagen membrane placement to completely cover the defect.

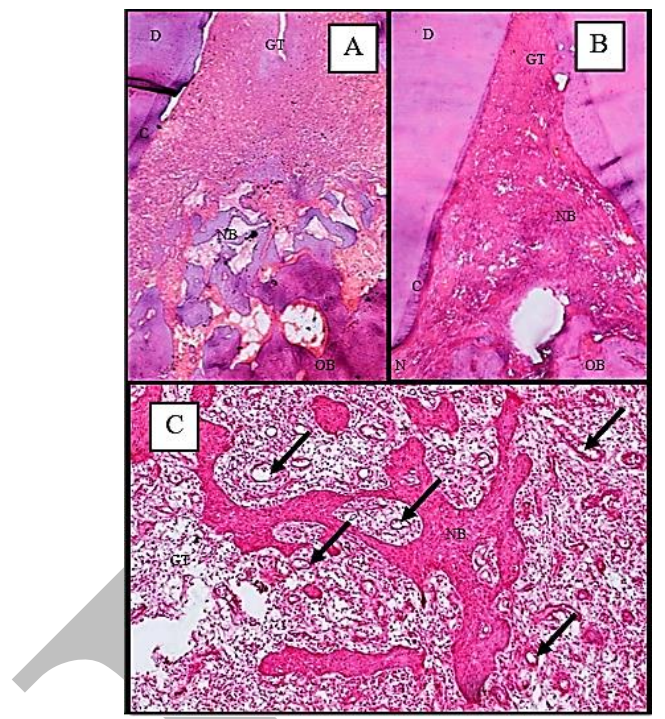

Figure (4): A, Light micrograph (LM) of control group at one month, showing old bone (OB) trabeculae at the base of the defect, covered by thin bone trabeculae of new bone (NB) extending towards the bifurcation area. Note: Dentin (D), and Cementum (C) (H\&E x 32).

B, LM of study group at one month, showing the defect area and the notches $(\mathrm{N})$ at both sides of the roots. Thick new bone trabeculae $(\mathrm{NB})$ are seen growing with many osteocytes inside. (H\&E x 100).

C, LM of another specimen of the study group at one month, showing new forming bone specules (NB) embedded inside granulation tissue. Note: high vascularity is indicated by the presence of multiple blood vessels with entrapped RBCs (Black arrows). (H\&E X 200).

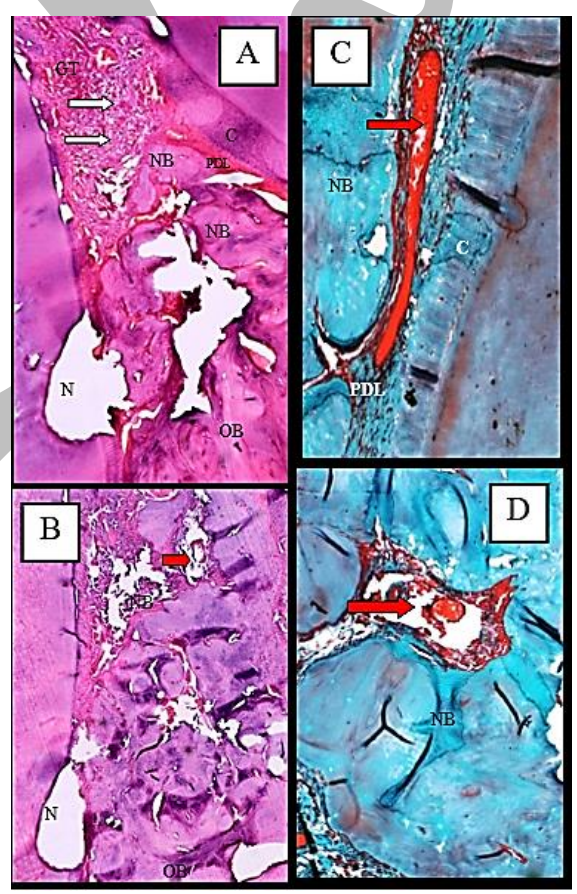

Figure (5): A, LM of control group at two months, showing growing new bone trabeculae (NB) extending coronal to the level of the notch $(\mathrm{N})$. Note: Old bone $(\mathrm{OB})$ apically and granulation tissue (GT) filling the rest of the defect with formation of small bone specules (White arrows) inside the GT. A good arrangement of (PDL) fibers with fibroblasts is found in between the NB and cementum (C) (H\&E X 32).

$B$, LM Study group at two months, showing the defect area nearly filled with trabeculae of $\mathrm{NB}$ formation merging with the $\mathrm{OB}$ at the base of the defect and growing towards the apex of the bifurcation area. Evidence of red bone marrow formation is seen (Red arrow).

C, LM another specimen from the same study group at two months, showing well formed PDL fibers, with the presence of fibroblasts, found between $\mathrm{C}$ and the NB. Note the high vascularity is seen by the presence of a wide blood vessel (Red arrow) with RBCS. (Trichrome X 200).

$D, L M$ of another specimen from the same study group at two months, showing NB nearly filling the defect area note the presence of vascular marrow spaces (Red arrows) formation filled with RCBs. (Trichrome $\mathbf{X}$ 100). 


\section{DICUSSION}

In this study, Grade II critical sized furcation defects were created in 4 mongrel dogs. A split mouth design was carried out in which the defects of the control side of each dog were augmented with $\beta$-TCP alloplast material and covered with a collagen membrane, while in the study side of the same dog the defects were augmented with a mixture of PRF cuts and $\beta$-TCP material covered with a PRF membrane then completely covered with a collagen membrane. After one and two months, tissue blocks containing the studied teeth with the surrounding soft tissue were obtained and examined histologically.

PRF was cut and mixed with $\beta$-TCP particles to be applied inside the created defects then covered by PRF membrane; collagen membrane was placed to completely cover the augmented defect. This technique of PRF application was also carried out by Bölükbaşı et al; (10) to maximize the contact between the defect walls and PRF from inside and from outside in order to maximize the effect of PRF. Although Bölükbaşı et al; (10) did not cover their created defects with collagen membranes we utilized collagen membranes in both groups to standardize the study approach, so any histological changes observed in either group will be attributed mainly to the role of the applied material only.

An important consideration during the use of PRF membrane was that it must be applied as early as possible after its separation and formation to ensure the maximum release of growth factors to the surgical site (11) and the produced exudate after the squeezing of the membrane can be used to hydrate the grafting material as it can be an additional source of growth factors, vitronectin and fibronectin (8)

One clinical advantage of PRF material that appeared during this study was its adhesive property. This kept the particles of $\beta$-TCP together attaching them tightly to the walls of the cavity, also the PRF membrane placed was adherent to the collagen membrane. This adherence is thought to provide strong stabilization of the membrane which is a prerequisite for successful GTR procedures for the prevention of downward growth of the epithelium. Similar to our findings Yilmaz et al; (12) and Kökdere et al; (13) observed that PRF accelerates the healing effect by keeping the particles of $\beta$-TCP and autogenous bone graft, respectively, together and attaching them tightly to the walls of the cavity by their adhesive properties favoring regeneration.

Platelets are nucleate cytoplasmic fragments containing spherical or oval granules with diameters ranging from 200 to $500 \mathrm{~nm}$. They form intracellular storage pools of proteins including platelet-derived growth factor (PDGF), transforming growth factor (TGF), vascular endothelial growth factors (VEGF) and insulin-like growth factor (IGF) and others. Secretion of these proteins happen after the fusion of the granules with the platelet cell membrane after platelet activation; these proteins later bind to the transmembrane receptors of the target cells causing the repair or regeneration mechanisms to start (14). Thus, it is believed that platelet concentrates can aid in a more predictable outcome when added to graft materials during various regenerative procedures (15).

In this study the histological results showed a distinct difference between the control and the study groups, regarding vascularization. The study group revealed numerous blood vessels rich in RBCs in the granulation tissue of the specimens at one month and also at 2 months more blood vessels and red bone marrow were observed.

Uchida et al; (1) stated that angiogenesis "the formation of new blood vessels inside the wound" is crucial in the delivery of oxygen, nutrients, and crucial cells from nearby tissues in the hypoxic microenvironment of healing wounds, they also stated that vascular endothelial growth factors (VEGF) were found responsible for regulating angiogenesis in the healing of surgically induced holes in the bone of rats. This was in accordance with similar results obtained by Steinbrech et al; (16) as they also observed the crucial role of VEGF in angiogenesis during the healing of long bones.

Yoon et al; (17) also observed that after the immunostaining of VEGF in regenerating cranial defects in rabbits, VEGF intensity was consistently higher in the study group; in which PRF was used, than in the control group at all age points, leading to more red bone marrow formation. They concluded that these findings suggest the ability of PRF in promoting angiogenesis by the secretion of VEGF.

Our study also lies in accordance with a study conducted by Choukroun et al; (18) in which they observed that PRF plays a role in enhancing cellular proliferation in the early stages of healing only, but it was found effective in the revascularization of the graft by supporting angiogenesis when combined with freeze-dried bone allografts during sinus augmentation procedures reducing the time needed prior to implant placement.

From here we can conclude the positive effect of PRF on angiogenesis, thus the vascularization of the graft aiding in its survival and promoting the regenerative process.

\section{CONCLUSION}

Within the limitations of our study and according to our findings we can conclude that the addition of PRF to bone grafting material with guided tissue regeneration procedures shows a favorable effect as it facilitates the vascularization of the defect space, facilitating cellular events that are favorable for periodontal regeneration.

\section{CONFLICT OF INTEREST}

The authors declare that they have no conflicts of interest.

\section{REFERENCES}

1. Uchida S, Sakai A, Kudo H, Otomo H, Watanuki M, Tanaka $\mathrm{M}$ et al. Vascular endothelial growth factor is expressed along with its receptors during the healing process of bone and bone marrow after drill-hole injury in rats. Bone.2003;32: 491-501.

2. Wang CJ, Chen HS, Chen CE, Yang KD. Treatment of nonunions of long bone fractures with shock waves. Clin Orthop Relat Res. 2001; 95:101-9.

3. Wang CJ, Huang KE, Sun YC, Yang YJ, Ko JY, Weng LH et al. VEGF modulates angiogenesis and osteogenesis in shockwave-promoted fracture healing in rabbits. J Surg Res. 2011;171: 114- 9.

4. Davi G, Patrono C. Platelet activation and atherothrombosis. N Engl J Med. 2007; 357:2482-94.

5. Mehta D, Deshpande N, Dave D, Modi B, Bharwani A. Platelet rich fibrin: New treatment modality in Grade II furcation defects. J Oral Dis Market. 2016; 1:1-5. 
6. Dohan DM, Choukroun J, Diss A, Dohan SL, Dohan AJ, Mouhyi $\mathrm{J}$ et al. Platelet-rich fibrin (PRF): A secondgeneration platelet concentrate. Part I: Technological concepts and evolution. Oral Surg Oral Med Oral Pathol Oral Radiol Endod. 2006; 101:37-44.

7. Dohan DM, Choukroun J, Diss A, Dohan SL, Dohan AJ, Mouhyi $J$ et al. Platelet-rich fibrin (PRF): a secondgeneration platelet concentrate. Part II: platelet related biologic features. Oral Surg Oral Med Oral Pathol Oral Radiol Endod. 2006; 101:45-50.

8. Toffler M, Toscano N, Holtzclaw D, Del Corso M, Ehrenfest DD. Introducing Choukroun's platelet rich fibrin (PRF) to the reconstructive surgery, The Journal of Implant and Advanced Clinical Dentistry. 2009;6: 21-32.

9. Bowers GM, Schallhorn RG, McClain PK, Morrison GM, Morgan R, Reynolds MA. Factors influencing the outcome of regenerative therapy in mandibular class II furcations: Part I. J Periodontol 2003; 74:1255-1268.

10. Bölükbaşı N, Yeniyol S, Tekkesin MS, Altunatmaz K. The Use of Platelet-Rich Fibrin in Combination with Biphasic Calcium Phosphate in the Treatment of Bone Defects: A Histologic and Histomorphometric Study. Current Therapeutic Research, Clinical and Experimental. 2013; 75:15-21.

11. Su CY, Kuo YP, Tseng YH, Su CH, Burnouf T. In vitro release of growth factors from platelet-rich fibrin (PRF): a proposal to optimize the clinical applications of PRF. Oral Surg Oral Med Oral Pathol Oral Radiol Endod. 2009;108: 56-61.

12. Yilmaz D, Dogan N, Ozkan A, Sencimen M, Oral BE, Mutlu I. Effect of platelet rich fibrin and beta tricalcium phosphate on bone healing.A histological study in pigs. Acta Cirúrgica Brasileira. 2014; 29:59-68.

13. Kökdere N, Baykul T, Findik Y. The use of platelet-rich fibrin (PRF) and PRF-mixed particulated autogenous bone graft in the treatment of bone defects: An experimental and histomorphometrical study. Dent Res J (Isfahan). 2015;12: 418-24.

14. Prakash S, Thakur A. Platelet concentrates: Past, present and future. J Maxillofac Oral Surg. 2011; 10:45-9.

15. Sunitha RV, Munirathnam NE. Platelet-rich fibrin: Evolution of a second-generation platelet concentrate Indian. J Dent Res. 2008;19: 42-6.

16. Steinbrech DS, Mehrara BJ, Saadeh PB, Greenwald JA, Spector JA, Gittes GK et al. VEGF expression in an osteoblast-like cell line is regulated by a hypoxia response mechanism. Am J Physiol Cell Physiol. 2000; 278: 853-60.

17. Yoon JS, Lee SH, Yoon HJ. The influence of platelet-rich fibrin on angiogenesis in guided bone regeneration using xenogenic bone substitutes: A study of rabbit cranial defects, Journal of Cranio-Maxillo-Facial Surgery. 2014; 34:1-7.

18. Choukroun J, Diss A, Simonpieri A, Girard MO, Schoeffler C, Dohan SL et al: Platelet rich fibrin (PRF): a secondgeneration platelet concentrate. Part V: histologic evaluations of PRF effects on bone allograft maturation in sinus lift. Oral Surg Oral Med Oral Pathol Oral Radiol Endod. 2006;101: 299-303. 